\title{
Time-resolved study of laser emission in nitrogen gas pumped by two near IR femtosecond laser pulses
}

\author{
Rostyslav Danylo ${ }^{1,2}$, Guillaume Lambert ${ }^{1}$, Yi LiU $^{2,3}$, Vladimir TikhonchuK ${ }^{4,5}$, Aurélien \\ HOUARD ${ }^{1}$, AND ANDRÉ MYSYROWICZ ${ }^{1,}$ \\ ${ }^{1}$ Laboratoire d'Optique Appliquée, ENSTA Paris, CNRS, Ecole Polytechnique, Institut Polytechnique de Paris, 828 Boulevard des Maréchaux, 91762 Palaiseau \\ cedex, France \\ ${ }^{2}$ Shanghai Key Lab of Modern Optical System, University of Shanghai for Science and Technology, 516, Jungong Road, 200093 Shanghai, China \\ ${ }^{3}$ CAS Center for Excellence in Ultra-intense Laser Science, 201800, Shanghai, China \\ ${ }^{4}$ University of Bordeaux-CNRS-CEA, CELIA, UMR 5107, 33405 Talence, France \\ ${ }^{5}$ ELI-Beamlines, Institute of Physics, Czech Academy of Sciences, 25241 Dolní Břežany, Czech Republic \\ *Corresponding author: andre.mysyrowicz@ensta-paris.fr
}

Compiled March 12, 2021

The time profile of the lasing signal at $391.4 \mathrm{~nm}$ emitted by a weakly ionized gas of nitrogen molecules at low pressure is measured under double excitation with intense femtosecond laser pulses at $800 \mathbf{~ n m}$. An abrupt decrease of the emission occurs at the time of arrival of the second pulse. It is explained by a transfer of population from ground to first excited ionic level and by a disruption of coherence, terminating the conditions for lasing in a V-scheme without population inversion.

http://dx.doi.org/10.1364/ao.XX.XXXXXX

\section{INTRODUCTION}

The laser emission from nitrogen gas pumped by an intense femtosecond laser pulse is a fascinating, highly controversial subject of study. More than 150 publications have been published on this question over the last few years, yet the origin of the lasing is still not settled. When an intense near IR femtosecond laser pulse irradiates a chamber filled with air or nitrogen gas, an under-dense plasma column is formed. With a linearly polarized incident laser pulse of intensity in the range $10^{14} \mathrm{~W} / \mathrm{cm}^{2}$ with central wavelength at $800 \mathrm{~nm}$, the emission from this plasma along the column axis consists of intense narrow lines at 391.4 or $427.8 \mathrm{~nm}$ [1-3], contrasting with the transverse luminescence from the plasma where these lines are buried in a multilevel spectrum [4]. These two wavelengths correspond to transitions between levels $\mathrm{B}^{2} \Sigma_{u}^{+}(0)$ and $\mathrm{X}^{2} \Sigma_{g}^{+}(0,1)$ of the singly ionized nitrogen molecule, where index $(0,1)$ refers to the vibrational quantum number. The emission at $391.4 \mathrm{~nm}$ appears at low gas pressure, reaches a peak around 50 mbar and disappears above 150 mbar, at which pressure the emission at $427.8 \mathrm{~nm}$ has started to appear and persists up to normal pressure [2]. Below $p \approx 100$ mbar, the emission at $391.4 \mathrm{~nm}$ can be amplified by orders of magnitude by injecting a weak femtosecond seed pulse at the same wavelength in the plasma column $[1,5]$. Amplification occurs even when the seed pulse is delayed by up to $10 \mathrm{ps}$, much longer than the pump pulse duration, showing that electronic excitation energy is stored in the plasma column, ready to be delivered. For this reason, the emission process has been called "cavity free lasing".

Three scenarios have been proposed to explain the $391.4 \mathrm{~nm}$ lasing: a first scenario assumes that a population inversion is established by the pump pulse between initial level $\mathrm{B}^{2} \Sigma_{u}^{+}(0)$ (hereafter denoted $B$ ) and final level $X^{2} \Sigma_{g}^{+}(0)$ (hereafter $X$ ) of the transition [6-8]. According to this model, this inversion is due to a fast depletion of population in $X$ level by a pump-induced transfer to intermediate ionic level $\mathrm{A}^{2} \Pi_{u}(2)$ (hereafter $A$ ), due to a quasi-resonance of the $X-A$ transition with the pump photon energy. A second model attributes the optical gain to a transient inversion of rotational population between levels $B$ and $X$ following pump pulse induced partial alignment of nitrogen molecules $[9,10]$. This amplification scheme does not require electronic population inversion between $B(0)$ and $X(0)$. Finally, a third model assumes no electronic nor rotational population inversion $[11,12]$. According to this model, lasing occurs because of interference between amplitudes of transition probabilities in a V-scheme arrangement. The scenarios with no electronic population inversion are especially interesting since by upgrading the photon energy from pump to emission, they offer a venue for amplifiers in the X-ray or $\Gamma$-ray domain, where population inversion is difficult to achieve [13-16]. Note that the second scenario requires molecules as an active medium while scenario 3 should also function with atoms.

The purpose of this manuscript is to discuss the origin of the lasing at $391.4 \mathrm{~nm}$, based on a study of the temporal shape of the laser emission at $391.4 \mathrm{~nm}$ following excitation by a sequence of 
two $800 \mathrm{~nm}$ pulses. The study is focused on the low pressure regime (30-50 mbar) where the seed induced amplification is highest. As will be discussed, the observed strong quenching of lasing [17-19] is consistent with V-model but cannot be easily explained by the other scenarios.

\section{EXPERIMENTAL SETUP}

The principle of the experiments is shown in Figure 1 (a detailed description of the set-up is given in the Supplementary Material). The output from a Ti:Sapphire laser emitting pulses at $800 \mathrm{~nm}$ at a repetition rate of $100 \mathrm{~Hz}$ with up to $15 \mathrm{~mJ}$ energy per pulse of $45 \mathrm{fs}$ duration is divided in 4 arms. A pump pulse (1) is sent in a gas chamber filled with nitrogen at a pressure of $30 \mathrm{mbar}$ and generates the plasma column. It is followed by a weaker control pulse (2) at $800 \mathrm{~nm}$ of adjustable delay. Pulses (1) and (2) have a diameter of $15 \mathrm{~mm}$ FWHM and are focused with a $\mathrm{f}=40 \mathrm{~cm}$ lens (L1) into the gas chamber. The energy of the control pulse is adjusted to obtain maximal quenching (see Figure 2 of Reference [17]) [20]. For each (1)-(2) delay, the lasing signal at $391.4 \mathrm{~nm}$ emerging from the plasma channel in the direction of the pump pulse is time resolved by mixing it in a nonlinear $\beta$-Barium Borate (BBO) crystal with pulse (4) and measuring the resulting sum frequency spectrum around $261 \mathrm{~nm}$ as a function of delay (1)-(4) with a high resolution spectrograph. The experiments are performed in the absence (Figure 2) and in the presence (Figure 3) of an orthogonally polarized seed pulse at $391 \mathrm{~nm}$ (3) injected about $300 \mathrm{fs}$ after the pump pulse (1). The $300 \mathrm{fs}$ pump/seed delay optimizes the lasing signal at $391.4 \mathrm{~nm}$ for this pump/seed polarization configuration.

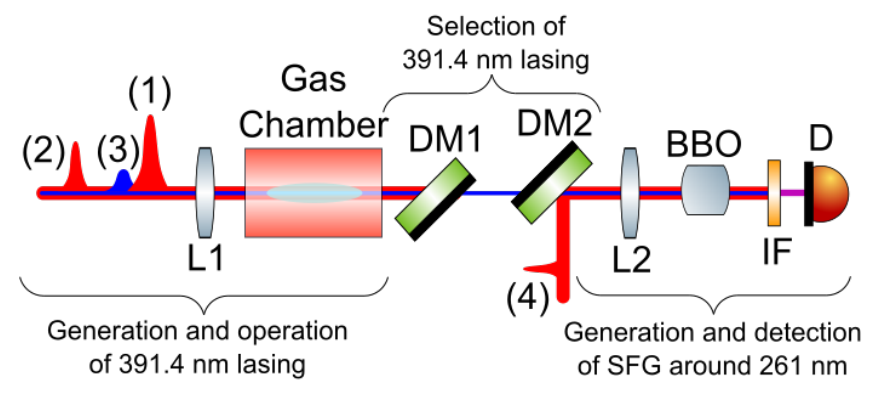

Fig. 1. Simplified experimental setup. $800 \mathrm{~nm}$ pump pulse (1), $800 \mathrm{~nm}$ control pulse (2) and $391 \mathrm{~nm}$ seed pulse (3) are collinearly recombined and focused with lens L1 in a gas chamber filled with nitrogen gas. Pump pulse (1) forms a plasma column where seed pulse (3) can be efficiently amplified while control pulse (2) serves to obliterate the lasing process. Dichroic mirror DM1 serves to filter the $800 \mathrm{~nm}$ laser emission and to transmit the amplified $391.4 \mathrm{~nm}$ lasing signal. It is recombined by another dichroic mirror DM2 with scan pulse (4) at $800 \mathrm{~nm}$. They are focused in a BBO crystal by lens L2 where Sum Frequency Generation (SFG) process takes place. All the emission except SFG around $261 \mathrm{~nm}$ is cut by interference filter IF. SFG signal is finally recorded by detection system D consisting in a spectrograph and associated CCD camera (for more details see Supplementary Material).

\section{RESULTS}

Let us consider first the case without external seed. In the absence of a control pulse (Figure 2a and black curve in Figure 2d), (a)

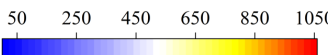

emission wavelength $(\mathrm{nm})$ $\begin{array}{lllll}386.9 & 389.1 & 391.4 & 393.6 & 395.9\end{array}$

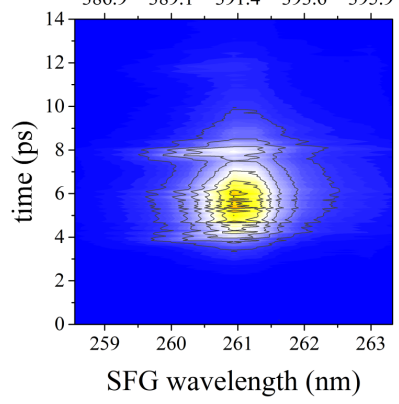

$\begin{array}{llllll}50 & 250 & 450 & 650 & 850 & 1050\end{array}$

(c)

emission wavelength $(\mathrm{nm})$ $\begin{array}{lllll}386.9 & 389.1 & 391.4 & 393.6 & 395.9\end{array}$

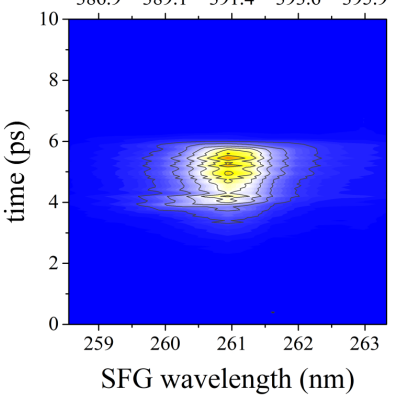

(d)

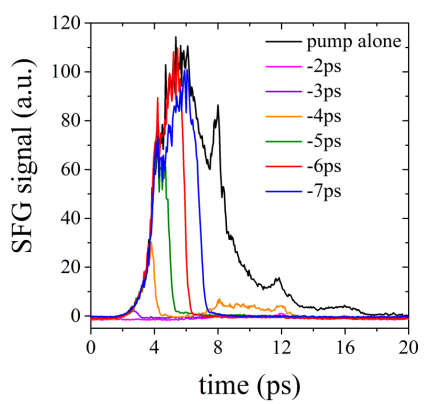

Fig. 2. Time-resolved lasing emission at $391.4 \mathrm{~nm}$ obtained with different pump/control delays in the absence of an external seed. Nitrogen gas pressure is $30 \mathrm{mbar}$, pump and control pulse energies are $2.1 \mathrm{~mJ}$ and $1.2 \mathrm{~mJ}$ respectively. a) Spectrogram recorded without control pulse. b) Spectrogram recorded with control pulse delayed by 5 ps. c) Spectrogram recorded with control pulse delayed by 6 ps. d) Wavelength integrated lasing signal for pump/control delays as indicated in the legend. Delays (1)-(2) are labelled negative to emphasize that control pulse (2) arrives after pump pulse (1).

the lasing starts after 3-4 ps, reaches a peak around 6 ps and persists beyond $15 \mathrm{ps}$. We also note the appearance of sharp peaks at 8,12 and (less resolved) 4 ps. The period of these peaks corresponds exactly to the period of rotation of ionized molecules in level $B[21,22]$. When the control pulse is injected, an abrupt decrease of the lasing emission occurs (Figure $2 b, c$ and $d$ ). The instant of suppression corresponds to the delay (1)-(2) between pump and control pulses. The same suppression effect occurs in the presence of a seed pulse (Figures 3 and 4). The main difference between Figures 2 and 3 is the shorter duration of the more intense lasing signal obtained with external seed, which now reaches its peak after $\sim 2$ ps [5]. Finally, Figure 5 shows the spectrum of the $391.4 \mathrm{~nm}$ emission obtained in the absence of external seed with a control pulse delay of $4.5 \mathrm{ps}$.

\section{DISCUSSION}

We now discuss these results by successively adopting the three different models. They exclude in our view the population inversion model. With a pump pulse of 1-2 mJ energy, we are in 
$\begin{array}{llllll}50 & 475 & 900 & 1325 & 1750 & 2175\end{array}$

(a)

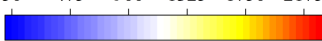

emission wavelength $(\mathrm{nm})$ $\begin{array}{lllll}386.9 & 389.1 & 391.4 & 393.6 & 395.9\end{array}$

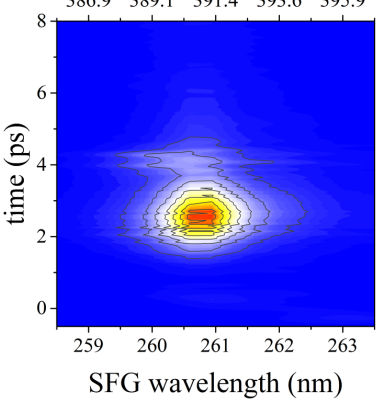

$\begin{array}{llllll}50 & 325 & 600 & 875 & 1150 & 1425\end{array}$

(c)

emission wavelength $(\mathrm{nm})$ $\begin{array}{lllll}386.9 & 389.1 & 391.4 & 393.6 & 395.9\end{array}$

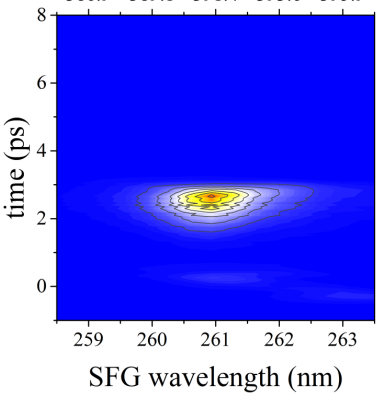

(d)

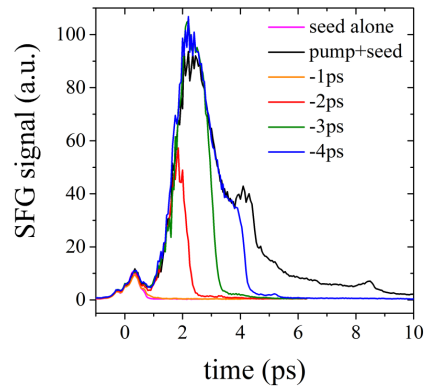

Fig. 3. Time-resolved lasing emission at $391.4 \mathrm{~nm}$ obtained with different pump/control delays in the presence of an external seed. Seed pulse (3) is injected $\sim 300 \mathrm{fs}$ after pump pulse (1). Nitrogen gas pressure is 30 mbar, pump and control pulse energies are $1.4 \mathrm{~mJ}$ and $0.8 \mathrm{~mJ}$ respectively. a) Spectrogram recorded without control pulse. b) Spectrogram recorded with control pulse delayed by 2 ps. The signal at $263 \mathrm{~nm}$ (shown by a red arrow) is a measurement artifact. It corresponds to a 2nd harmonic component generated in the $\mathrm{BBO}$ crystal and upconverted by the scan pulse (4). c) Spectrogram recorded with control pulse delayed by $3 \mathrm{ps}$. Spurious signal at $263 \mathrm{~nm}$ is not clearly observed because the stronger signal at $261 \mathrm{~nm}$ requires less integration time for the detection. d) Wavelength integrated temporal profile of $391.4 \mathrm{~nm}$ lasing for pump/control delays as indicated in the legend.

a situation where gain is not saturated, as shown in Figure S2 of the supplementary material. This means that an increase of the pump pulse intensity, or alternatively injection of an additional pulse at $800 \mathrm{~nm}$ in the immediate wake of the pump pulse, should further increase population inversion and consequently lead to an increase of the lasing signal. Figures 2 and 3 show the opposite effect: instead of an increase, there is an abrupt and near total suppression of the lasing emission.

The second model predicts narrow gain windows occurring when inversion of rotation population between $B$ and $X$ ions is accomplished. The appearance of sharp peaks in the emission profile is in principle compatible with this model. However, according to Reference [9], their appearance period should not correspond exactly to the period of revival of $\mathrm{N}_{2}^{+}$molecules in $B$ state, as observed in Figure 2. Also, the continuous component of the lasing at other delays is not explained, nor the suppression of the lasing signal by a control pulse.

The third model accounts well for the results. As discussed in References [11] and [12], a large amplification can occur in the presence of long-lived coherent polarizations $X-B$ and $X-A$ coupling $B$ to $A$ via $X$ in a V-scheme arrangement. Amplification requires population inversion between $B$ and $A$ but no inversion of population between $B$ and X. Simulations based on Maxwell-Bloch equations yield a delay for the appearance of the amplified signal, in agreement with the experiments [5, 11, 23]. Note that the sharp peaks are not reproduced in the simulations because the model does not include the formation of coherent rotational wavepackets. The quenching of the lasing signal upon injection of a control pulse is also explained. As it was the case in Reference [17] we interpret this observation by the V-scheme model of laser without population inversion. The new aspect in the present work is that we also present a simulation of the effect. This simulation shows that in addition to the population transfer the destruction of the A-X coherence induced by the control pulse also contributes to the quenching effect. The numerical simulation consists in four steps: (i) modeling of the Nitrogen gas ionization and ionic excitation by the pump pulse; (ii) evolution of the $391 \mathrm{~nm}$ signal with a A-X coherence maintained by a post-pulse [24]; (iii) perturbation of plasma by the control pulse and re-excitation of $\mathrm{N}_{2}^{+}$; (iv) evolution of the $391 \mathrm{~nm}$ signal from the re-excited Nitrogen ions (for more simulation details see Supplementary Materials). The abrupt drop-down of lasing shown in Figures 2 and 3 is well-reproduced in simulation, as shown in Figure 4.

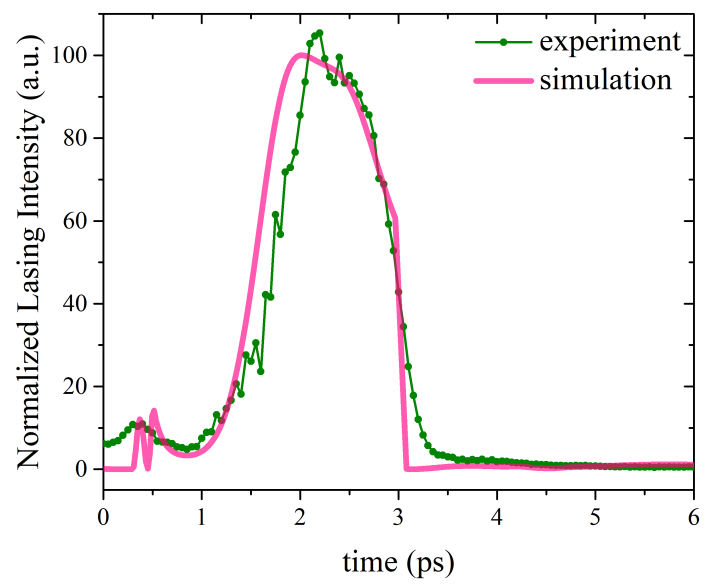

Fig. 4. Comparison of measured (green line and circles) and calculated (pink curve) temporal profiles of lasing at $391.4 \mathrm{~nm}$, quenched by a 3 ps delayed $800 \mathrm{~nm}$ control pulse. Experimental curve is the same as in Figure $3 d$. In the simulations, the $800 \mathrm{~nm}$ pump pulse intensity is $2.6 \times 10^{14} \mathrm{~W} / \mathrm{cm}^{2}$, while the control pulse intensity is $1.0 \times 10^{14} \mathrm{~W} / \mathrm{cm}^{2}$. The external seed pulse of intensity $2 \times 10^{8} \mathrm{~W} / \mathrm{cm}^{2}$ is injected $300 \mathrm{fs}$ after the pump pulse. Post-pulse maintaining $X-A$ coherence in the system is approximated by an exponential function with $6 \times 10^{9} \mathrm{~W} / \mathrm{cm}^{2}$ initial amplitude, 7 ps decay time, detuning between post-pulse and $X-A$ transition frequencies is $1.1 \mathrm{ps}^{-1}$ [24]. Gain is calculated along $1 \mathrm{~cm}$ plasma column with Nitrogen gas pressure of 30 mbar. 
It is interesting to note that a pump/control delay of $4.5 \mathrm{ps}$ opens a brief time window bounding a revival of a coherent rotation wavepacket of $B$ molecules. The emission spectrum around $391.45 \mathrm{~nm}$, shown in Figure 5, corresponds to the $\mathrm{P}$ branch $B(\mathrm{~J}) \rightarrow X(\mathrm{~J}+1)$ of the $B-X$ transition, where $\mathrm{J}$ is the total angular momentum of a molecule. It can be decomposed into a narrow line and a broader component. The duration corresponding to the broader component is on the order of $250 \mathrm{fs}$, in good agreement with the duration (Full Width at Half Maximum) of the alignment half-cycle of $B$ molecules $[9,25,26]$ while the narrow component corresponds to a longer-lived emission from non-aligned molecules. A similar broadening of the $\mathrm{R}$ branch $B(\mathrm{~J}) \rightarrow X(\mathrm{~J}-1)$, is also observed. A detailed study of the line broadening will be the subject of a forthcoming manuscript.

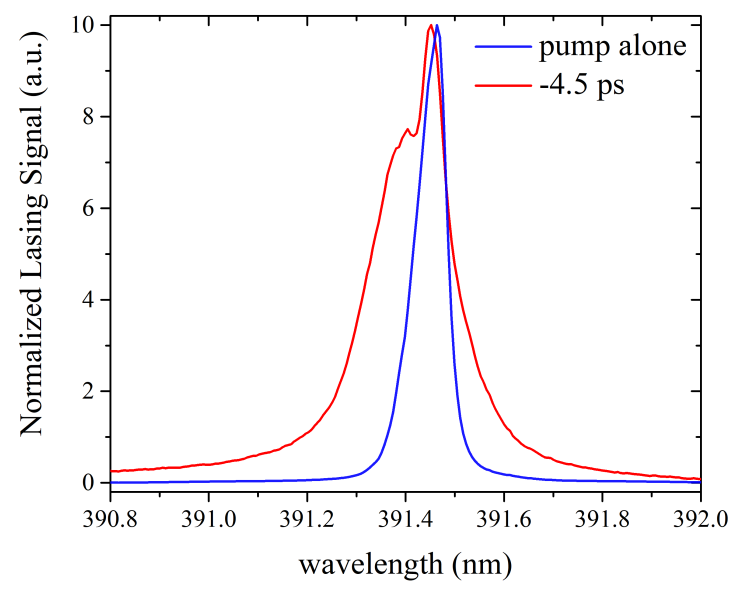

Fig. 5. Normalized spectra of self-seeded lasing, P-branch. Blue curve corresponds to the case when only $800 \mathrm{~nm}$ pump pulse arrives, red curve is recorded with a control pulse retarded by 4.5 ps. Experimental conditions are the same as in Figure 2. Delay (1)-(2) is labelled negative to emphasize that control pulse (2) arrives after pump pulse (1)

\section{CONCLUSION}

To summarize, time-resolved measurements of the emission profile obtained in a nitrogen gas at low pressure with a sequence of two $800 \mathrm{~nm}$ pump pulses reveal an abrupt cut of the lasing signal upon arrival of the second pulse. This effect is explained in the frame of the V-model by a transition from the ground level $\mathrm{X}^{2} \Sigma_{g}^{+}(0)$ of the lasing to an intermediate level $\mathrm{A}^{2} \Pi_{u}(2)$ and by a disruption of the coherences induced by the second pulse, terminating the condition for amplification $n_{B}>n_{A}$. Based on these results, we conclude that the important gain observed at $391.4 \mathrm{~nm}$ at low pressure corresponds to an amplification process without population inversion. We emphasize that this conclusion is valid for a nitrogen gas at low pressure pumped by a femtosecond pump pulse centered at $800 \mathrm{~nm}$, of peak intensity in the range of a few $10^{14} \mathrm{~W} / \mathrm{cm}^{2}$. It is possible that other mechanisms set in at other pump wavelengths, or higher pump pulse intensities, or higher gas pressures.

Funding. Project EPAT3 from Direction Générale de l'Armement (DGA). Project LQ1606 from the Czech National Programme of Sustainability II. Innovation Program of Shanghai Municipal
Education Commission (Grant No. 2017-01-07-00-07-E00007), Shanghai Municipal Science and Technology Commission (No. 17060502500).

Disclosures. The authors declare no conflicts of interest.

\section{REFERENCES}

1. J. Yao, G. Li, C. Jing, B. Zeng, W. Chu, J. Ni, H. Zhang, H. Xie, C. Zhang, H. Li, H. Xu, S. L. Chin, Y. Cheng, and Z. Xu, New J. Phys. 15, 023046 (2013).

2. Y. Liu, Y. Brelet, G. Point, A. Houard, and A. Mysyrowicz, Opt. Express 21, 22791 (2013).

3. T.-J. Wang, J. Ju, J.-F. Daigle, S. Yuan, R. Li, and S. L. Chin, Las. Phys. Lett. 10, 125401 (2013).

4. A. Talebpour, S. Petit, and S. L. Chin, Opt. Commun. 171, 285 (1999).

5. Y. Liu, P. Ding, G. Lambert, A. Houard, V. Tikhonchuk, A. Mysyrowicz, Phys. Rev. Lett. 115, 133203 (2015).

6. H. Xu, E. Lötstedt, A. Iwasaki, and K. Yamanouchi, Nat. Commun. 6, 8347 (2015).

7. J. Yao, S. Jiang, W. Chu, B. Zeng, C. Wu, R. Lu, Z. Li, H. Xie, G. Li, C. Yu, Z. Wang, H. Jiang, Q. Gong, and Y. Cheng, Phys. Rev. Lett. 116, 143007 (2016).

8. H. Li, M. Hou, H. Zang, Y. Fu, E. Lötstedt, T. Ando, A. Iwasaki, K. Yamanouchi, and H. Xu, Phys. Rev. Lett. 122, 013202 (2019).

9. M. Richter, M. Lytova, F. Morales, S. Haessler, O. Smirnova, M. Spanner, and M. Ivanov, Optica 7, 586 (2020).

10. M. Lytova, M. Richter, F. Morales, O. Smirnova, M. Ivanov, and M. Spanner, Phys. Rev. A 102, 013111 (2020).

11. A. Mysyrowicz, R. Danylo, A. Houard, V. Tikhonchuk, X. Zhang, Z. Fan, Q. Liang, S. Zhuang, L. Yuan, and Y. Liu, APL Photon. 4, 110807 (2019).

12. V. Tikhonchuk, Y. Liu, R. Danylo, A. Houard, and A. Mysyrowicz, New J. Phys. https://doi.org/10.1088/1367-2630/abd8bf.

13. O. A. Kocharovskaya, Ya. I. Khanin, JETP Lett. 48, 630 (1988).

14. S.E. Harris, Phys. Rev. Lett. 62, 1033 (1989).

15. M. O. Scully, M. Fleischhaurer, Science 263, 337 (1994).

16. A. Svidzinsky, L. Yuan, and M. O. Scully, New J. Phys. 15, 053044 (2013).

17. R. Danylo, G. Lambert, Y. Liu, V. Tikhonchuk, A. Houard, and A. Mysyrowicz, Opt. Lett. 45, 4670 (2020).

18. M. Britton, M. Lytova, D. H. Ko, A. Alqasem, P. Peng, D. M. Villeneuve, C. Zhang, L. Arissian, and P. B. Corkum, Phys. Rev. A 102, 053110 (2020).

19. A. Zhang, M. Lei, J. Gao, C. Wu, Q. Gong, and H. Jiang, Opt. Express 2714922 (2019).

20. With a weaker control pulse a more complicated situation with partial quenching is observed, the discussion of which is outside the scope of this paper.

21. L. Klynning and P. Pages, Phys. Scr. 25, 543 (1982).

22. G. Herzberg, Molecular Spectra and Molecular Structure: Volume I Spectra of Diatomic Molecules., 2nd ed. (Krieger Publishing Company, Florida USA, 1989).

23. A. Zhang, Q. Liang, M. Lei, L. Yuan, Y. Liu, Z. Fan, X. Zhang, S. Zhuang, C. Wu, Q. Gong, and H. Jiang, Opt. Express 27, 12638 (2019).

24. Recently we have carried out experiments on lasing at $391.4 \mathrm{~nm}$ pumped with a high contrast $\left(10^{-10}\right) 800 \mathrm{~nm}$ laser pulse of $10 \mathrm{fs}$ duration and a peak intensity in the range of $5 \times 10^{14} \mathrm{~W} / \mathrm{cm}^{2}$. Amplification of externally injected seed was still observed, suggesting that the experimentally measured long lasting coherent polarization $A-X$ [11] may not be due to the presence of a laser post-pulse. We investigate alternative explanations for the origin of the long lasting $A-X$ polarization.

25. J. F. Ripoche, G. Grillon, B. Prade, M. Franco, E. Nibbering, R. Lange, and A. Mysyrowicz, Opt. Commun. 135, 310 (1997).

26. T. Seidman, J. Chem. Phys. 115, 5965 (2001). 


\section{REFERENCES}

1. J. Yao, G. Li, C. Jing, B. Zeng, W. Chu, J. Ni, H. Zhang, H. Xie, C. Zhang, H. Li, H. Xu, S. L. Chin, Y. Cheng, and Z. Xu,"Remote creation of coherent emissions in air with two-color ultrafast laser pulses," New J. Phys. 15, 023046 (2013).

2. Y. Liu, Y. Brelet, G. Point, A. Houard, and A. Mysyrowicz,"Self-seeded lasing in ionized air pumped by $800 \mathrm{~nm}$ femtosecond laser pulses," Opt. Express 21, 22791 (2013).

3. T.-J. Wang, J. Ju, J.-F. Daigle, S. Yuan, R. Li, and S. L. Chin, "Selfseeded forward lasing action from a femtosecond Ti:sapphire laser filament in air," Las. Phys. Lett. 10, 125401 (2013).

4. A. Talebpour, S. Petit, and S. L. Chin, "Re-focusing during the propagation of a focused femtosecond Ti:sapphire laser pulse in the air," Opt. Commun. 171, 285 (1999).

5. Y. Liu, P. Ding, G. Lambert, A. Houard, V. Tikhonchuk, A. Mysyrowicz, "Recollision-induced superradiance of ionized nitrogen molecules," Phys. Rev. Lett. 115, 133203 (2015).

6. H. Xu, E. Lötstedt, A. Iwasaki, and K. Yamanouchi, "Sub-10-fs population inversion in $\mathrm{N}_{2}^{+}$in air lasing through multiple state coupling," Nat. Commun. 6, 8347 (2015).

7. J. Yao, S. Jiang, W. Chu, B. Zeng, C. Wu, R. Lu, Z. Li, H. Xie, G. Li, C. Yu, Z. Wang, H. Jiang, Q. Gong, and Y. Cheng, "Population redistribution among multiple electronic states of molecular nitrogen ions in strong laser fields," Phys. Rev. Lett. 116, 143007 (2016).

8. H. Li, M. Hou, H. Zang, Y. Fu, E. Lötstedt, T. Ando, A. Iwasaki, K. Yamanouchi, and $\mathrm{H}$. Xu, "Significant enhancement of $\mathrm{N}_{2}^{+}$lasing by polarization-modulated ultrashort laser pulses," Phys. Rev. Lett. 122, 013202 (2019).

9. M. Richter, M. Lytova, F. Morales, S. Haessler, O. Smirnova, M. Spanner, and M. Ivanov, "Rotational Quantum Beat Lasing Without Inversion," Optica 7, 586 (2020).

10. M. Lytova, M. Richter, F. Morales, O. Smirnova, M. Ivanov, and M. Spanner, " $\mathrm{N}_{2}^{+}$Lasing: Gain and Absorption in the Presence of Rotational Coherence," Phys. Rev. A 102, 013111 (2020).

11. A. Mysyrowicz, R. Danylo, A. Houard, V. Tikhonchuk, X. Zhang, Z. Fan, Q. Liang, S. Zhuang, L. Yuan, and Y. Liu, "Lasing without population inversion in $\mathrm{N}_{2}^{+}$," APL Photon. 4, 110807 (2019).

12. V. Tikhonchuk, Y. Liu, R. Danylo, A. Houard, and A. Mysyrowicz, "Theory of femtosecond strong field ion excitation and subsequent lasing in $\mathrm{N}_{2}^{+}$," New J. Phys. https://doi.org/10.1088/1367-2630/abd8bf.

13. O. A. Kocharovskaya, Ya. I. Khanin, "Coherent amplification of an ultrashort pulse in a three-level medium without a population inversion," JETP Lett. 48, 630 (1988).

14. S.E. Harris, "Lasers without inversion: Interference of lifetimebroadened resonances," Phys. Rev. Lett. 62, 1033 (1989).

15. M. O. Scully, M. Fleischhaurer, "Lasers without inversion," Science 263, 337 (1994).

16. A. Svidzinsky, L. Yuan, and M. O. Scully, "Transient lasing without inversion," New J. Phys. 15, 053044 (2013).

17. R. Danylo, G. Lambert, Y. Liu, V. Tikhonchuk, A. Houard, and A. Mysyrowicz, "Quantum erasing of laser emission in $\mathrm{N}_{2}^{+}$," Opt. Lett. 45, 4670 (2020).

18. M. Britton, M. Lytova, D. H. Ko, A. Alqasem, P. Peng, D. M. Villeneuve, C. Zhang, L. Arissian, and P. B. Corkum, "Control of $\mathrm{N}_{2}^{+}$air lasing," Phys. Rev. A 102, 053110 (2020).

19. A. Zhang, M. Lei, J. Gao, C. Wu, Q. Gong, and H. Jiang, "Subfemtosecond-resolved modulation, of superfluorescence from ionized nitrogen molecules by $800-n m$ femtosecond laser pulses," Opt. Express 2714922 (2019).

20. With a weaker control pulse a more complicated situation with partial quenching is observed, the discussion of which is outside the scope of this paper.

21. L. Klynning and P. Pages, "The Band Spectrum of $\mathrm{N}_{2}^{+}$," Phys. Scr. 25, 543 (1982).

22. G. Herzberg, Molecular Spectra and Molecular Structure: Volume I Spectra of Diatomic Molecules., 2nd ed. (Krieger Publishing Company, Florida USA, 1989).

23. A. Zhang, Q. Liang, M. Lei, L. Yuan, Y. Liu, Z. Fan, X. Zhang, S. Zhuang,
C. Wu, Q. Gong, and H. Jiang, "Coherent modulation of superradiance from nitrogen ions pumped with femtosecond pulses," Opt. Express 27, 12638 (2019).

24. Recently we have carried out experiments on lasing at $391.4 \mathrm{~nm}$ pumped with a high contrast $\left(10^{-10}\right) 800 \mathrm{~nm}$ laser pulse of $10 \mathrm{fs}$ duration and a peak intensity in the range of $5 \times 10^{14} \mathrm{~W} / \mathrm{cm}^{2}$. Amplification of an externally injected seed was still observed, suggesting that the experimentally measured long lasting coherent polarization $A-X[11]$ may not be due to the presence of a laser post-pulse. We investigate alternative explanations for the origin of the long lasting $A-X$ polarization.

25. J. F. Ripoche, G. Grillon, B. Prade, M. Franco, E. Nibbering, R. Lange, and A. Mysyrowicz, "Determination of the time dependence of $n_{2}$ in air," Opt. Commun. 135, 310 (1997).

26. T. Seidman, "On the dynamics of rotationally broad, spatially aligned wave packets," J. Chem. Phys. 115, 5965 (2001). 